\title{
CONSERVACIÓN Y MANTENIMIENTO DE LA MEMORIA: UN APORTE DE SALUD MENTAL AL ENVEJECIMIENTO ACTIVO
}

\author{
Carlos E. Rodríguez A. MD*, Ángela Tatiana Puentes**
}

\section{Resumen}

La creciente población de ancianos del país nos plantea el reto de asegurar su calidad de vida. Como alternativa importante la Organización Mundial de la Salud propone el enfoque del envejecimiento activo. El área de salud mental de la Facultad de Medicina de la Fundación Universitaria de Ciencias de la Salud realiza un proyecto con un grupo de ancianos de la comunidad de la localidad de Los Mártires dirigido a la conservación y mantenimiento de la memoria dentro de la perspectiva del envejecimiento activo.

Palabras clave: ancianos, envejecimiento activo, reserva cognoscitiva, funcionamiento cerebral, memoria. Abreviaturas: EA, envejecimiento activo.

\section{MEMORY CONSERVATION AND MAINTENANCE: A CONTRIBUTION OF MENTAL HEALTH TO ACTIVE AGING}

\begin{abstract}
The growing number of older persons in our country poses the challenge of ensuring their quality of life. The World Health Organization proposes an active aging approach as an important alternative. The mental health area of the School of Medicine of the Fundación Universitaria de Ciencias de la Salud is conducting a project with an elderly group from the Los Mártires neighborhood, focused on memory conservation and maintenance within the active aging perspective.
\end{abstract}

Key words: the elderly, active aging, cognitive reserve, brain function, memory.

Fecha recibido: septiembre 20 de 2010 - Fecha aceptado: octubre 21 de 2010

* Jefe de la División Biopsicosocial, Profesor Titular de Psiquiatría, Facultad de Medicina, Fundación Universitaria de Ciencias de la Salud. Bogotá DC. Colombia.
** Interna de la Rotación Electiva de Psiquiatria. Fundación Universitaria de Ciencias de la Salud. Hospital de San José. Bogotá DC. Colombia. 


\section{Introducción}

Un logro extraordinario del siglo XX a nivel mundial fue el aumento de la expectativa de vida al nacer en casi treinta años. A comienzos del siglo XXI existían en el planeta 580 millones de personas de sesenta años o más, y se prevé que serán más de mil millones en 2020 . Esto supondrá un aumento de $75 \%$ en este grupo de edad frente a un incremento inferior al 50\% de la población mundial en conjunto. El 70\% de esta población mayor vivirá en los países en desarrollo. ${ }^{1}$ El envejecimiento de la población latinoamericana no tiene precedente; a Estados Unidos y a Canadá les tomó más de sesenta años pasar del 7\% al 14\% de adultos mayores, mientras que en América Latina y el Caribe será sólo 25 años o menos.

En cuanto a Colombia, en un siglo los habitantes pasaron de 4'355.470a 41'468.384, de los cuales 6,3\% (2'612.508) son mayores de 65 años y de éstos el 54,6\% pertenece al sexo femenino. El $75 \%$ de la población general vive en las cabeceras municipales, a pesar de que en áreas rurales hay mayores tasas de fecundidad, lo que se traduciría en un incremento natural de la población allí ubicada, pero el efecto es contrarrestado por las altas tasas de migración y desplazamiento. ${ }^{2}$

Colombia es uno de los países de América Latina con transformaciones intensas en su estructura poblacional por el proceso de transición demográfica. Su acelerado crecimiento se convierte en un escenario caracterizado por un rápido incremento de la población mayor de 60 años, la cual hapasado de 600.000 en 1950 a tres millones en 2001 y será de quince y medio millones en 2050. Está pasando de ser un país de niños a una nación progresivamente vieja. En 1959 la relación existente de tres menores de cinco años por cada mayor de sesenta, será la opuesta en 2050 cuando habrá en promedio tres mayores de sesenta años por cada menor de cinco años. ${ }^{3}$

Del total de la población adulta mayor, el $63,12 \%$ se concentra en Boyacá, Tolima, Cundinamarca, Caldas, Antioquia, Valle del Cauca, Quindío, Santander, Atlántico y Bolívar. Además, el $28,8 \%$ se encuentra en las principales ciudades: Bogotá, Medellín, Cali y Barranquilla.
En Bogotá residen 564.223 personas de sesenta años y más, que representan el $15,2 \%$ de la población colombiana en este grupo de edad y el $20,2 \%$ de las personas mayores residentes en las cabeceras de las ciudades, alcanzando una tasa anual de crecimiento de 5,3\%, superior a la de este grupo poblacional a nivel nacional en 1.2 puntos porcentuales. Este significativo incremento de la población mayor de sesenta años evidencia el proceso de envejecimiento de la población colombiana y en particular la que vive en Bogotá. La concentración de personas mayores en las diferentes localidades de la ciudad es la siguiente: las altas concentradas están en Engativá (13,3\%), Suba (12,5\%) y Kennedy (11,9\%); las medianas en Usaquén (8,2\%), San Cristóbal (5,4\%), Barrios Unidos (5,4\%), Ciudad Bolívar (5,3\%), Rafael Uribe Uribe (5,3\%), Puente Aranda (5\%), Bosa (4,9\%), Fontibón $(4,2 \%)$ y Teusaquillo $(3,5 \%)$; y las menores en Chapinero (2,9\%), Tunjuelito (2,8\%), Usme (2,7\%), Antonio Nariño $(2,2 \%)$, Los Mártires $(1,9 \%)$, Santa Fe $(1,8 \%)$ y La Candelaria $(0,5 \%){ }^{4}$

Según proyecciones del DANE hasta el 2015, de una población estimada para el 2009 de 97.283 habitantes para la localidad de Los Mártires, 11.845 serán mayores de sesenta años (12,2\%). Frente a esta creciente población anciana el siglo XXI tiene el reto de asegurar su calidad de vida. Para países como el nuestro será doble: de una parte, se debe continuar la lucha contra las enfermedades infecciosas, la desnutrición y la mortalidad infantil, mientras de otra se enfrentará al rápido crecimiento de las enfermedades crónicas.

\section{¿Qué estamos haciendo?}

A finales de 1990 la Organización Mundial de la Salud, partiendo del reconocimiento de los derechos humanos de las personas mayores y los principios de las Naciones Unidas de independencia, participación, dignidad, cuidado y autorrealización, propone el enfoque del envejecimiento activo como un proceso de optimización de las oportunidades de bienestar físico, social y mental durante toda la existencia, con el objetivo de ampliar la esperanza de vida saludable, la productividad y su calidad en la vejez. ${ }^{5}$ La palabra activo incluye la apropiación de esta perspectiva por parte del sujeto y su implicación continua 
en los aspectos sociales, económicos, culturales y cívicos de su entorno.

\section{Determinantes del envejecimiento activo}

La satisfacción de la persona que envejece en forma activa depende de una diversidad de factores "determinantes" que lo rodean a él, a su familia y al país. Estos factores son el género y la cultura, los sistemas sanitario y social, los económicos como son los ingresos, trabajo y protección social; los del entorno físico; los personales comportamentales y del ambiente social.

La cultura comoelemento supraestructural influye sobre los otros determinantes del envejecimiento. A pesar de haber completado el proceso de transición epidemiológica que ha convertido al país en una población de adultos mayores, sigue imperando una cultura de la juventud en la cual alcanzada la tercera década se es ya viejo. De otra parte se considera al anciano como dependiente e improductivo. Esto afecta en especial a las mujeres, por tradición excluidas del empleo calificado y con bajos ingresos, cuya mayor expectativa de vida los hace un grupo mayoritario que desde el punto de vista económico es vulnerable. Todo esto propicia la creación de un estereotipo cultural que considera la vejez como un proceso evolutivo deteriorante, en el cual tanto el cuerpo como la mente van perdiendo el orden y la integridad y llevan de manera inexorable a grados variables de incapacidad. ${ }^{6}$

Por fortuna esta concepción estereotipada comienza a cambiar gracias al conocimiento aportado por investigaciones recientes que apoyan la propuesta de un EA al señalar como factores importantes un buen funcionamiento cerebral, la predisposición genética, el estilo y la calidad de vida, un adecuado afrontamiento del estrés, la participación social y las redes sociales.

\section{Predisposición genética}

Los aspectos genéticos constituyen el elemento inmodificable del envejecimiento que da lugar al llamado envejecimiento primario, es intrínseco al organismo y consiste en un deterioro en los procesos fisiológicos como consecuencia de la edad. Son todos los cambios progresivos, deletéreos y universales que experimenta el organismo con el paso del tiempo, que se inician con la concepción y finalizan con la muerte. Es un proceso irreversible que afecta en forma heterogénea las células que conforman los seres vivos. Los factores restantes constituyen el envejecimiento secundario, derivado de los efectos de la enfermedad, el trauma y el estrés, sobre los cuales es posible desarrollar intervenciones preventivas. $^{7}$

\section{Funcionamiento cerebral}

Desde el punto de vista psiquiátrico el funcionamiento cerebral es un elemento clave pues determina la calidad de la memoria y con ella la identidad del sujeto que envejece, además de las funciones de relación y funcionalidad motora. El envejecimiento se acompaña de una visible reducción de la capacidad de crear y evocar nuevas memorias, de disminución sensible de los períodos de concentración y más aún, de un enlentecimiento de la velocidad de procesamiento de la información. La revisión de diversos estudios que miden las diferencias según la edad en el desempeño de pruebas neuropsicológicas, establece que la disminución normal de la capacidad cognoscitiva en individuos de setenta años varía entre el $15 \%$ y $40 \%$ de los puntajes crudos, dependiendo de la función y la prueba utilizada. La educación es un factor importante que puede llevar a un mayor uso de las redes cerebrales, lo que se manifestaría como una habilidad para afrontar el daño cerebral sin un déficit cognoscitivo visible. $^{8}$

Stern propuso el concepto de reserva cognoscitiva entendido como la ausencia de síntomas en presencia de cambios neuropatológicos. Es el resultado de la interacción de la reserva biológica que corresponde al desarrollo sano del cerebro y la plasticidad cerebral, entendida como la capacidad del mismo para adaptarse a la presencia de daño cerebral. Está influenciada del nacimiento a la muerte por los mismos factores señalados antes al referimos al éxito del EA.

El déficit cognoscitivo relacionado con la del envejecimiento puede ser la expresión de la alteración de regiones cerebrales específicas y su expresión 
sintomática e intensidad dependerán de la vulnerabilidad individual, que no es otra cosa que la reserva cognoscitiva que posea el sujeto anciano. El proceso de envejecer es complejo y dentro de la perspectiva actual se caracteriza por la reorganización, optimización y utilización de la plasticidad cerebral para alcanzar la adaptación y adecuación al entorno y lograr un EA.

La reserva cognoscitiva se ha definido operativamente como un modelo tipo "umbral" en el que ésta tendría un papel de protección frente a las lesiones. El modelo reconoce la existencia de diferencias individuales en la capacidad de reserva y la existencia de un nivel de umbral crítico. Una vez la reserva cerebral ha disminuido por debajo de dicho nivel, aparecería el déficit.

Según lo han señalado estudios como el de "Desarrollo del Adulto" de Harvard los factores psicosociales juegan un papel igual o más importante que la genética en la protección de esta reserva. Se han encontrado como factores protectores y garantes de un EA los siguientes:

- No fumar ni abusar del alcohol.

- Relación emocional estable.

- Relaciones sociales significativas.

- Ejercicio físico regular.

- Dieta saludable.

- Estimulación intelectual permanente.

- Actividades placenteras.

- Afrontar nuevos retos.

- Visión optimista.

- Afrontamiento adecuado de las situaciones estresantes.

- Prevención temprana de enfermedades cardiovasculares.

\section{Respuesta a la propuesta del envejecimiento activo}

La cátedra de psiquiatría dirigida a estudiantes de décimo semestre de la Facultad de Medicina de la Fundación
Universitaria de Ciencias de la Salud ha desarrollado una línea gerontopsiquiátrica, dentro de la cual un foco especial de interés ha sido el de la memoria. El seminario Aspectos Emocionales del Envejecimiento como parte del curso regular de psiquiatría, nos llevó desde hace tres años a proyectarlo a la comunidad de la localidad de Los Mártires. De la revisión teórica cerrada de los temas, pasamos a su divulgación y socialización con los ancianos usuarios de los centros Lafayette y Refugio de la Secretaría Distrital de Integración Social.

El fundamento teórico de este seminario parte de la consideración de la vejezcomo la etapa final del desarrollo humano dentro del modelo psicosocial propuesto por Erik Erikson. Se espera que la persona que durante las siete etapas previas delmodelo ha resuelto en forma adecuada el conflicto propio de cada una de ellas y alcanzado el logro de la misma, llegará a la vejez con el bagaje de mecanismos de defensa del yo de altísimo valor energético, como son el altruismo y el humor. Esto le permitirá afrontar con éxito el conflicto antitético integridad vs. desesperación y alcanzar el logro de la octava y última etapa que es la sabiduría. Quienes no lo logran estarán desesperados y presentarán en forma sintomática la evidencia de una psicopatología: ansiosa, depresiva, hipocondríaca, somatizadora o paranoide que puede llegar hasta la psicosis franca. El éxito, además de los factores psicológicos señalados, dependerá de la riqueza del entorno social en el cual se desarrollan las personas. ${ }^{9}$

Los ancianos que asisten a estos centros de la Secretaría de Integración Social de la Alcaldía Menor de Los Mártires afrontaron a lo largo de su vida difíciles condiciones psicosociales, por tal razón sorprende que el nivel de psicopatología no sea el esperado. Es probable que se deba al interés de participación social que muestran al asistir a las actividades de estos centros, el cual constituye un factor protector, porque como lo señala Fernando Savater ${ }^{10}$, la política entendida como la participación activa en la vida de la ciudad, en este caso de la localidad, es uno de los dos remedios simbólicos frente a la muerte, el otro es la religión.

A partir de las reflexiones originadas en la divulgación de los aspectos teóricos del seminario señalado antes y 
de la intención de lograr el desarrollo de los factores protectores del EA en el interior de los grupos de ancianos, derivamos en la propuesta del proyecto denominado: Mantenimiento y conservación de la memoria. Tienecomo objetivo general optimizar la utilización de la reserva cognoscitiva de los ancianos dentro de la perspectiva del envejecimiento activo. Como objetivos específicos están la estimulación del funcionamiento cerebral de los ancianos participantes del proyecto y el desarrollo de relaciones sociales significativas.

Durante los períodos académicos del 2008 al 2010, los estudiantes han concurrido los miércoles por la mañana al centro Lafayette ubicado en el barrio Eduardo Santos y los viernes al centro El Refugio en el barrio Santa Fe. Han participado en forma activa en el desarrollo las actividades informativas y de entrenamiento cerebral. En la actualidad se encuentran en actividad treinta y cuatro ancianos con un alto nivel de participación y adherencia.

La rentabilidad social suele parecer siempre menor frente a la económica en los proyectos sociales, pero creemos que en este corto tiempo hemos impactado de manera positiva la mentalidad tanto de los ancianos como de los jóvenes que se aproximaron con interés genuino al fenómeno del envejecimiento.

\section{Referencias}

1. Kalache A. Active ageing makes the difference. Bull World Health Orga. 1999; 77(4): 299.

2. Bogotá. Alcaldia Mayor. Secretaria Distrital de Salud. Lineamientos plan de intervenciones colectivas: promoción de la calidad de vida y salud de envejecimiento y vejez[monografia en Internet]. Bogotá:Alcaldía Mayor; 2008. [citado 2011 feb. 8 ]. Disponible en: http://190.25.230.149:8080/ dspace/bitstream/123456789/282/I/Lineamientos\%20P IC\% 20Envejecimiento\%20y\%20Vejez\%202009.pdf

3. Ramirez Zuñiga JA. Situación actual de las personas adultas mayores en Colombia, legislación y programas de atención al adulto mayor [monografia en Internet]. Neiva : Universidad Surcolombiana. Facultad de Enfermeria; 2009. [citado 2011 feb. 8 ]. Disponible en: http://www.scribd.com/doc/ 20297780/Situacion-Actual-de-La-Persona-Adulta-Mayor-en-Colombia

4. DANE. Proyecciones Municipales de población 1985- 2020 sexo y grupo de edad. [monografia en Internet]. Bogotá : DANE; 2005. [citado 2011 feb. 8 ]. Disponible en: http://www.dane.gov.co/daneweb_V09/index.php?option= com_content\&view $=$ article $\&$ id $=75 \&$ Itemid $=72$

5. Pajares R. A propósito del envejecimiento activoy de la II Asamblea Mundial sobre el Envejecimiento: que estamos haciendo y que nos queda por hacer. Aten Primaria. 2002 Jun; 30 (2): 77-79.

6. Gaviria M, Gaviria N. Vejez, creatividady cerebro. Avances Psiquiatr.biol. 2008; 9: 38-55.

7. Rodriguez CE, Téllez JE. Gerontopsiquiatria. En: Toro RJ, Yepes LE, Palacio JE, Téllez JE, editores. Fundamentos de medicina: Psiquiatria. Medellin : Corporación para Investigaciones Biológicas; 2010. p. 463-4.

8. Roe CM, Xiong Ch, Miller JP, Morris JC. Education andAlzheimer disease without dementia. Support for the cognitive reserve hypothesis. Neurology. 2007;68: 223-28.

9. Erikson E. La adultez. México: Fondo de Cultura Económica; 1981.p. 14-57

10. Savater F. Política para Amador. Barcelona : Editorial Ariel; 1992. 